\title{
A COUNTEREXAMPLE FOR COMMUTATION IN TENSOR PRODUCTS OF $C^{*}$-ALGEBRAS
}

\author{
R. J. ARCHBOLD
}

\begin{abstract}
An example is given to show the failure of the analogue for $C^{*}$-algebras
\end{abstract} of the commutation theorem for von Neumann tensor products.

Let $A, B, C, D$ be $C^{*}$-algebras with $A \subseteq C$ and $B \subseteq D$. Tomiyama [10, p. 29] has raised the question as to whether $(A \otimes B)^{c}=A^{c} \otimes B^{c}$. In this context $\otimes$ denotes the spatial tensor product and $(A \otimes B)^{c}$ (respectively $\left.A^{c}, B^{c}\right)$ is the relative commutant of $A \otimes B$ (respectively $A, B)$ in $C \otimes D$ (respectively $C, D$ ). It is easy to see that the inclusion $A^{c} \otimes B^{c} \subseteq(A \otimes B)^{c}$ is always valid. In the special case where $C$ has an identity $1, A=C 1$ and $B=D$, the question has an affirmative answer [7, Theorem 1], and the result has been generalized to the case of an arbitrary $C^{*}$-tensor norm [1], [4]. It is therefore tempting to conjecture that the question has an affirmative answer at least in the case where $A=\mathrm{Cl}$ (but $B$ is an arbitrary $C^{*}$-subalgebra of $D$ ). In this note we present a counterexample based on results of Choi [5], Wassermann [13] and Voiculescu [11].

We begin by recalling some facts about slice maps [9]. Let $A$ and $B$ be $C^{*}$-algebras and let $\phi \in A^{*}$. The right slice map $R_{\phi}: A \otimes B \rightarrow B$ is the unique bounded linear mapping with the property that $R_{\phi}(a \otimes b)=\phi(a) b(a \in A, b \in$ $B)$. A triple $(A, B, J)$, where $J$ is a closed two-sided ideal of $B$, is said to verify the slice map conjecture [12] if whenever $x \in A \otimes B$ and $R_{\phi}(x) \in J$ for all $\phi \in A^{*}$ then $x \in A \otimes J$. It is well known that $(A, B, J)$ verifies the slice map conjecture if and only if $A \otimes J$ is the kernel of the canonical *-homomorphism $\mu: A \otimes B \rightarrow A$ $\otimes(B / J)$. This is because

$$
\text { ker } \mu=\left\{x \in A \otimes B \mid R_{\phi}(x) \in J \text { for all } \phi \in A^{*}\right\} \text {. }
$$

The following result is implicit in [13, 2.5]. Although we shall apply it in a rather special case, we state it in the given form since it may be of independent interest.

Proposition. Suppose that $B / J$ is a nuclear $C^{*}$-algebra. Then the triple $(A, B, J)$ verifies the slice map conjecture.

Proof. Suppose that $(A, B, J)$ does not verify the slice map conjecture. The canonical *-isomorphism of the algebraic tensor product $A \odot(B / J)$ into $(A \otimes B) /(A \otimes J)$ induces on $A \odot(B / J)$ a $C^{*}$-norm which is distinct from the least $C^{*}$-norm since ker $\mu \neq A \otimes J$. This contradicts the nuclearity of $B / J$.

Received by the editors April 4, 1980.

AMS (MOS) subject classifications (1970). Primary 46LOS.

Key words and phrases. $C^{*}$-algebra, tensor product, relative commutant, slice map, free group. 
REMARK. Since any quotient of a nuclear $C^{*}$-algebra is nuclear [6], it follows from the Proposition that $(A, B, J)$ verifies the slice map conjecture whenever $B$ is a nuclear $C^{*}$-algebra. It is shown in [2] that in fact it suffices to assume that $B$ is just a $C^{*}$-subalgebra of some nuclear $C^{*}$-algebra in order to conclude that $(A, B, J)$ verifies the slice map conjecture.

We now give the counterexample. Let $C=C^{*}\left(F_{2}\right)$, the full $C^{*}$-algebra of the free group on two generators, and let $J$ be the kernel of the canonical *-homomorphism from $C$ onto the $C^{*}$-algebra of the left regular representation of $F_{2}$. Since $C$ is separable it follows from [8, 3.7.5] that there is a faithful (nondegenerate) representation $\pi$ of $C$ on a separable Hilbert space $H$ (of infinite dimension). By [5, Corollary 2] $\pi(C)$ contains no nonzero compact operator. It follows that we may regard $C$ as a $C^{*}$-subalgebra of the Calkin algebra ( $D$ say) associated with $H$. Since $\pi$ was nondegenerate we may assume that the identity 1 of $D$ lies in $C$. Let $A=C 1$ and let $B=(J+C 1)^{c}$ (the relative commutant of $J+C 1$ in $\left.D\right)$. Then, with the relative commutants taken as indicated in the opening paragraph, we have the following result.

THEOREM. $(A \otimes B)^{c} \neq A^{c} \otimes B^{c}$.

Proof. Since $J+\mathrm{Cl}$ is a separable $C^{*}$-subalgebra of $D$ it follows from [11] (see also [3, p. 345]) that $(J+\mathbf{C l})^{c c}=J+\mathrm{Cl}$. Thus $B^{c}=J+\mathbf{C l}$ and so $A^{c} \otimes B^{c}=$ $C \otimes(J+C l)$. By $[13,2.7$, Remark] there exists $x \in C \otimes C(\subseteq C \otimes D)$ such that $x \notin C \otimes J$ and $R_{\phi}(x) \in J$ for all $\phi \in C^{*}$ (where $R_{\phi}$ is the right slice map $C \otimes C \rightarrow C$ associated with $\phi)$. Applying the Proposition to the triple $(C, J+$ $\mathrm{Cl}, J)$ we see that $x \notin C \otimes(J+\mathbf{C l})$.

Let $b \in B$. For $\phi \in C^{*}$ we have

$$
R_{\phi}[x(1 \otimes b)-(1 \otimes b) x]=R_{\phi}(x) b-b R_{\phi}(x)=0
$$

Hence $x(1 \otimes b)-(1 \otimes b) x=0[9$, Theorem 1], and so $x \in(A \otimes B)$.

The author is grateful to M.-D. Choi for providing a copy of [5] prior to publication.

\section{REFERENCES}

1. R. J. Archbold, On the centre of a tensor product of $C^{*}$-algebras, J. London Math. Soc. (2) 10 (1975), 257-262.

2. R. J. Archbold and C. J. K. Batty, C*-tensor norms and slice maps, J. London Math. Soc. (2) 22 (1980), 127-138.

3. W. B. Arveson, Notes on extensions of $C^{*}$-algebras, Duke Math. J. 44 (1977), 329-355.

4. C. J. K. Batty, On relative commutants in tensor products of $C^{*}$-algebras, Math. Z. 151 (1976), 215-218.

5. M-D. Choi, The full $C^{*}$-algebra of the free group on two generators, Pacific J. Math. (to appear).

6. M-D. Choi and E. G. Effros, Nuclear $C^{*}$-algebras and injectivity: the general case, Indiana Univ. Math. J. 26 (1977), 443-446.

7. R. Haydon and S. Wassermann, A commutation result for tensor products of $C^{*}$-algebras, Bull. London Math. Soc. 5 (1973), 283-287.

8. G. K. Pedersen, $C^{*}$-algebras and their automorphism groups, Academic Press, London, 1979. 
9. J. Tomiyama, Applications of Fubini type theorem to the tensor products of $C^{*}$-algebras, Tôhoku Math. J. (2) 19 (1967), 213-226.

10. $\longrightarrow$ Tensor products and approximation problems of $C^{*}$-algebras, Publ. Res. Inst. Math. Sci. 11 (1975), 163-183.

11. D. Voiculescu, A non-commutative Weyl-von Neumann theorem, Rev. Roumaine Math. Pures Appl. 21 (1976), 97-113.

12. S. Wassermann, The slice map problem for $C^{*}$-algebras, Proc. London Math. Soc. (3) 32 (1976), 537-559.

13. __ On tensor products of certain group C*-algebras, J. Funct. Anal. 23 (1976), 239-254.

Department of Mathematics, University of Aberdezn, Aberdeen AB9 2TY, Scotland 